\title{
Lattice Boltzmann Simulation of Heat and Mass Transfer in Solar-wall System
}

\author{
Yousheng $\mathrm{Xu}^{*}$, Jiugu Shao \\ 1 Department of Physics, Zhejiang Normal University, Jinhua, China \\ * Corresponding E-mails: ysyou@zjnu.cn
}

Keyword: LBM; solar wall; heat and mass transfer; porous medium.

\begin{abstract}
In this paper, the problem of heat and $m$ ass transfer in Solar-wall System is solve $d$ by lattice Boltzmann method. The lattice Boltzm ann equation is governed by the heat conduction equation. The heat transfer and flow characteristics for the Solar-wall System with air as heat transfer medium are simulated. It is found that the perfor mance parameters of the porous solar wall are influenced by the outlet air velocity and structure of the porous solar wall such as the porosity and the porous solar wall width.
\end{abstract}

\section{Introduction}

With the urbanization and new buildings accelerated, the demand of energy is increased. But the problems like the shortage of ener gy supply, power rationing, interruption of power supply have occurred in many large cities. The proportion of en ergy consumed by buildings has attained thirty percent in China. And fifty to sixty percent of building ener gy consumption is consumed by air-condition and heating [1].As concerning the indoor comfort requirements gradually increase, the building energy consumption in the proportion is increa sing at the same time. So it is a significant project that dealing with buildi ng energy consumption especially the air-condition and heating. As people found, it is the only way to deal with it pe rmanently that finding renewable ener gy instead the traditional ener gy [2]. The developm ent and application of new ener gy is em ergency, and application of solar energy is an effective way to alleviate this problem. On the other hand the solar energy is one of new energy which is of large scale application in present.

Double-skin glass curtain wall[3], Trombe Wall[4]and Solar house technology[5] have been used gradually. But the Solar -wall Systems which is ba sed on the new idea of heating fresh air of the building by solar ener gy having higher ef ficiency than traditional glass solar collectors is certified by U.S. Departm ent of Ener gy's National Rene wable Energy Laboratory [6]. Then the solar radiation absorbed is used to dem and for heating using conductivity, radiation and convection. Some heat-absorption walls were studied by researchers at home and abroad. Numerous researchers developed to explore the perform ance of heat transfer and flowing. The simulation of heat transfer in pores of heat collectors by CFD $m$ odel which is only focus on the heat transfer when the air through the pores by S.J.Arulanandam, K.GT ea'D,Hollands and E. Brundret t(1999)[7].It discusses five parameters which effect the heat exchange effectiveness for conditions of no wind. Simulations were carried out over a wide range of conditions, and the results are incorporated into a correlation model. Because of the no-wind assum ptions, the model is of lim ited direct use. Dymond and Kutscher[8] developed pipe network methods to analyze the airflow distribution. The model can run quickly on the personal com puter (PC). Previous research results on heat exchange effectiveness, pressure drop, and wind heat loss were incorpo rated. But the accuracy of the $m$ odel is uncertain when a short pipe system instead the cavity in $n$ egative pressure zone of real system. Based on the overall performance of solar colle ctor, paper[16] presented that CF D model is used to do research on the uniform ity of airflow on th e solar collector and found the key to guarantee the high heat transfer rate is keeping the heat uniformly,the uniformity of airflow would effect the uniformity of heat. Hollick [9-1 1] studied on the wind parallel to the solar collector . Wang Chongjie and He Wenjing do the experim ent research on solar collector system. Paper [12] $\mathrm{f}$ ound the porosity of solar wall combination have large influence on heat effect. And paper designed two kinds of porous 
solar wall with dif ferent ventilation type, calculate, analysis and comparison the character of heat transfer and flow in system by num erical simulate method. It found ventilation type af fects the temperature field and flow field of the heating room.

In this paper, based on solar -wall heat technology, by using the lattice Boltzmann method of analysis and simulation, factors which af fected the perform ance of the porous solar wall were discussed. Such as the outlet air velocity, porosity and the porous solar wall width were discussed respectively. According to the in teraction of buoyancy force and suction force, the outlet air velocity is the main factor to influen ce heat collecting efficiency. The porous solar wall width and the porosity are also to influence the heat transfer efficiency and heat collecting efficiency.

\section{Macroscopic equations}

Assuming that the Boussinesq limit holds, the governing equations of the heat and mass transfer in the solar wall can be described as follows:

$$
\begin{gathered}
\nabla \cdot \mathbf{u}=0 \\
\frac{\partial \mathbf{u}}{\partial t}+\mathbf{u} \cdot \nabla\left(\frac{\mathbf{u}}{\phi}\right)=-\frac{1}{\rho_{f}} \nabla \cdot(\phi P)+\nabla \cdot\left(v_{e} \nabla \mathbf{u}\right)+\mathbf{F} \\
\sigma \frac{\partial \mathbf{T}}{\partial t}+\mathbf{u} \cdot \nabla \mathbf{T}=\nabla\left(\alpha_{e} \nabla \mathbf{T}\right)
\end{gathered}
$$

where $\mathbf{T}, \mathbf{u}$, and $P$ are temperature, volume-averaged velocity and press, respectively. In the Eq. (3), the ratio between the heat cap acities of the pure substance and po rous medium is describ ed by $\sigma=\phi+(1-\phi) \rho_{s} C_{p s} / \rho_{f} C_{p f}$. Here $\sigma$ is unity, $\mathbf{F}$ is the total force and which can be described as

$$
\mathbf{F}=-\frac{\phi v}{K} \mathbf{u}-\frac{\phi F_{\phi}}{\sqrt{K}}|\mathbf{u}| \mathbf{u}+\phi \mathbf{G}
$$

where the first term is the inte raction force of $m$ aterial and porous m edium, the second term is the influence of geometric construction, and the last term is the body force

$$
\mathbf{G}=-g \beta\left(T-T_{0}\right), \quad K=\frac{\phi^{3} d^{2}}{150(1-\phi)^{2}}, \quad F_{\phi}=\frac{1.75}{\sqrt{150 \phi^{3}}}
$$

Here $\mathbf{G}, K, F_{\phi}$ representing the buoyancy force, the $\mathrm{p}$ ermeability and the geo metric function, respectively.

The governing equations can be described by non-dimensional parameters

$$
J=\frac{v_{e}}{v}, \quad D a=\frac{K}{L^{2}}, \quad \operatorname{Pr}=\frac{v}{\alpha_{e}}, \quad R a=\frac{g \beta \Delta T L^{3}}{v \alpha_{e}}
$$

\section{LBGK model for the velocity field.}

The modified lattice Boltzmann equation (LBE) which is used for porous flow can be described as

$$
f_{i}\left(\mathbf{x}+\mathbf{e}_{i} \cdot \Delta t, t+\Delta t\right)=f_{i}(\mathbf{x}, t)-\frac{1}{\tau}\left[f_{i}(\mathbf{x}, t)-f_{i}^{e q}(\mathbf{x}, t)\right]+\Delta t \cdot \mathbf{F}_{i}
$$

where $\mathbf{e}_{i}$ are the discrete ve locity directions, $\tau$ is the relaxation tim e. At present work, we choose the D2Q9 model, and the discrete velocities are given by

$$
\mathbf{e}_{i}=\left\{\begin{array}{c}
0, i=0 \\
\left(\cos \frac{(i-1) \pi}{2}, \sin \frac{(i-1) \pi}{2}\right), i=1 \sim 4 \\
\sqrt{2}\left(\cos \frac{(2 i-9) \pi}{4}\right), \sin \left(\frac{(2 i-9) \pi}{4}\right), i=5 \sim 8
\end{array}\right.
$$


$f_{i}^{(e q)}$ is the equilibrium distribution function (EDF), the EDF is defined as

$$
f_{i}^{e q}=\rho \omega_{i}\left[1+3(\mathbf{e} \cdot \mathbf{u})+4.5 \frac{(\mathbf{e} \cdot \mathbf{u})^{2}}{\phi}-1.5 \frac{(\mathbf{u} \cdot \mathbf{u})}{\phi}\right]
$$

With a view to the porous effect on other ex ternal force and gas flow, the force term $\mathbf{F}_{i}$ in Eq.(7) is given by

$$
\mathbf{F}_{i}=\rho \omega_{i}\left(1-\frac{1}{2 \tau}\right)\left[\frac{\mathbf{e}_{i} \cdot \mathbf{F}}{c_{s}^{2}}+\frac{\mathbf{u F}:\left(\mathbf{e}_{i} \cdot \mathbf{e}_{i}-c_{s}^{2} \mathbf{I}\right)}{\phi c_{s}^{4}}\right]
$$

With the weight $\omega_{0}=4 / 9, \quad \omega_{i}=1 / 9$ for $i=1 \sim 4, \quad \omega_{i}=1 / 36$ for $i=5 \sim 8$.

The velocity and volume density are defined as

$$
\mathbf{u}=\frac{\mathrm{v}}{c_{0}+\sqrt{c_{0}^{2}+c_{1}|\mathrm{v}|}}, \quad \rho=\sum_{i} f_{i}
$$

where $c_{0}, c_{1}$ and $\mathrm{v}$ in Eq. (11) are given by

$$
c_{0}=\frac{1}{2}\left(1+\phi \frac{\Delta t v}{2 K}\right), \quad c_{1}=\phi \frac{\Delta t F_{\phi}}{2 \sqrt{K}}, \quad \mathrm{v}=\sum_{i} \frac{e_{i}}{\rho}+\frac{\Delta t}{2} \phi \mathbf{G}
$$

To test and verify the LB Eq. (7), we utiliz e the Chapman-Enskog Expansion to deduce it, and then the Eqs. (1) (2)can be recovered as follows

$$
\begin{gathered}
\frac{\partial \rho}{\partial t}+\nabla \cdot(\rho \mathbf{u})=0 \\
\frac{\partial(\rho \mathbf{u})}{\partial t}+\nabla \cdot\left(\frac{\rho \mathbf{u} \mathbf{u}}{\phi}\right)=-\nabla p+\nabla\left[\rho v_{e}(\nabla \mathbf{u}+\mathbf{u} \nabla)\right]+\mathbf{F}
\end{gathered}
$$

where $p=c_{s}^{2} \rho / \phi$ is pressure, the viscosity is given by

$\nu_{e}=c_{s}^{2} \Delta t(\tau-0.5)$

Owing to the incompressible limit $\rho_{0}=\rho_{1}=$ const, Eqs. (13) $\sim(14)$ simplify to the governing Eqs. (1) $\sim(2)$.[13]

\section{LBGK model for the temperature field.[14]}

For the he at and $\mathrm{m}$ ass transfer in the porous s olar wall, the presen ce of the air $\mathrm{m}$ atrix will certainly affect the tem perature distribution function and its equilibrium. This influence should be incorporated in the LBE for the temperatu re field. Therefore, the energy Eq. (3) can be solved by the following LBE:

$$
T_{i}\left(\mathbf{x}+\mathbf{e}_{i} \cdot \Delta t, t+\Delta t\right)=T_{i}(\mathbf{x}, t)-\frac{1}{\tau^{\prime}}\left[T_{i}(\mathbf{x}, t)-T_{i}^{e q}(\mathbf{x}, t)\right]
$$

$T_{i}^{(e q)}$ is the equilibrium distribution function (EDF), the EDF is defined as follows:

$$
T_{i}^{e q}=\omega_{i} T\left(1+\frac{e_{i} \mathbf{u}}{c_{s}^{2}}\right)
$$

Through the Chapm an-Enskog multiscaling expansion technique, and then the E q.(3) can be recovered as follows

$$
\sigma \frac{\partial \mathbf{T}}{\partial t}+\mathbf{u} \cdot \nabla \mathbf{T}=\nabla\left(\alpha_{e} \nabla \mathbf{T}\right)
$$

The thermal conductivity is given by

$$
\alpha_{e}=\sigma c_{s}^{2} \Delta t\left(\tau^{\prime}-0.5\right)
$$

\section{Physical model}

A Solar wall System is belong to passive solar heating system s. The absorption surface with selective coating is designed to face the south, wh ich can converts solar energy into heat. It takes advantages of high heat ef ficiency,well-designed fresh air system [15],yield good econom ic 
returns[16],wide rage of applica tion and energy saving[17]. And the Solar-wall System consists of the porous solar wall which is always covered the outside of buildings' outer wall, a canopy overhanging the solar wall, exhaust fans that is used in exhausting the air of the interspaces between the porous solar wall and the wall, and ducting insi de the building for air di stribution. The scheme of the physics model is set for calcu lating easily in Fig. 1. In wint ertime, the outside air enters the cavity through pore in the daytime. During flowing, solar radiant heat absorbed by the solar wall is achieved, then in the heated $\mathrm{p}$ ressure the air en ter ventilation system and distributed to different room by ducting. At night, the heat which the wall lost is absorbed by the air in the cavity will be back under the fans working. In this way, not only keep the fresh air, but also supply the heat and make the wall be $h$ eat exchanger. However, during summer, the fans will be s topped, the hot air outside come in through the bottom of the solar wall and come out from the upper, the heat should not enter in the indoor [18-20].

\section{Numerical Results and Discussion}

Fig.2. shows that the air stream lines in the po rous solar wall sy stem. It can be clearly seen that the streamlines become dense, and the air veloc ity increases along the vert ical direction of the porous solar wall, which is com patible with previous literature well and it is prove $d$ that LBM is reliable. Obviously, the position of the fan has $r$ emarkable influence on flow characteristics and the heat transfer of the porous solar wall. So, the fa $\mathrm{n}$ should be fixed properly to undertake fully heat transfer between the air and the porous solar wall, a nd increase the thermal efficiency of the porous wall.

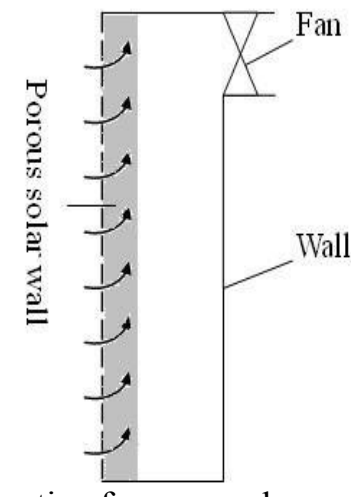

Fig. 1 Schematic of porous solar wall system

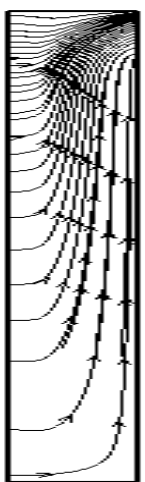

Fig. 2 Streamline for the porous solar wall system

Outlet air velocity is the main factor to influence the heat transfer of the porous wall. In order to study the influence of flow velocity, two parts are discussed: (1) the heat transfer ef ficiency under the different outlet air velocity; (2) the heat co llecting efficiency under the dif ferent outlet air velocity.
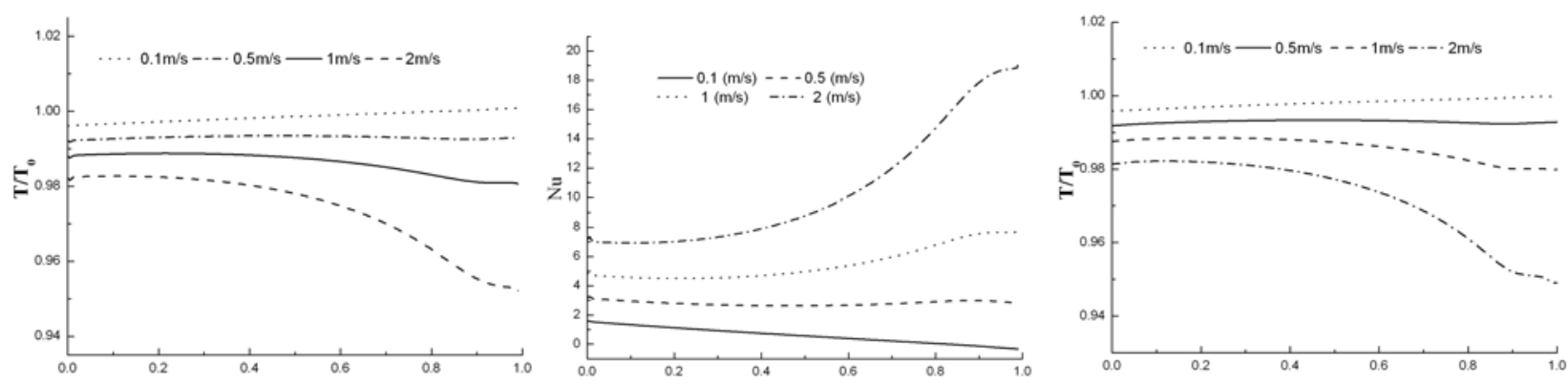

(a) Temperature of the porous solar wall (b) Nusselt number of the porous solar wall(c) Temperature of the pipe

Fig. 3 Effect of the outlet air velocity on the of the Solar-wall System alone the vertical direction the pipe

Fig. 3 shows the tem perature comparison and Nusselt number along the vertical line at the porous solar wall and the pipe respectively. With low outlet air velocity, the porous solar wall has good thermal uniformity, and heat efficiency is increasing will higher out let air velocity. When 
outlet air ve locity is high, the porous solar wall has bad therm al uniformity, in upper area heat efficiency is higher and in lower area heat efficiency is lower. To prevent thermal un-uniformity, the appropriate outlet air velocity should be given.

Fig. 4 shows the inlet air velocity and airflow ra te of the porous solar wall alone the vertical direction under the different outlet air velocity respectively. With high outlet air velocity, the porous solar wall has bad therm al uniformity, in upper area heat efficiency is higher and in lower area heat ef ficiency is lower. When the outlet air velocity is low, the porous solar wall has good thermal uniformity, but through porous solar wall ha ppened. To prevent reverse flow, the outlet air velocity can not be too small.
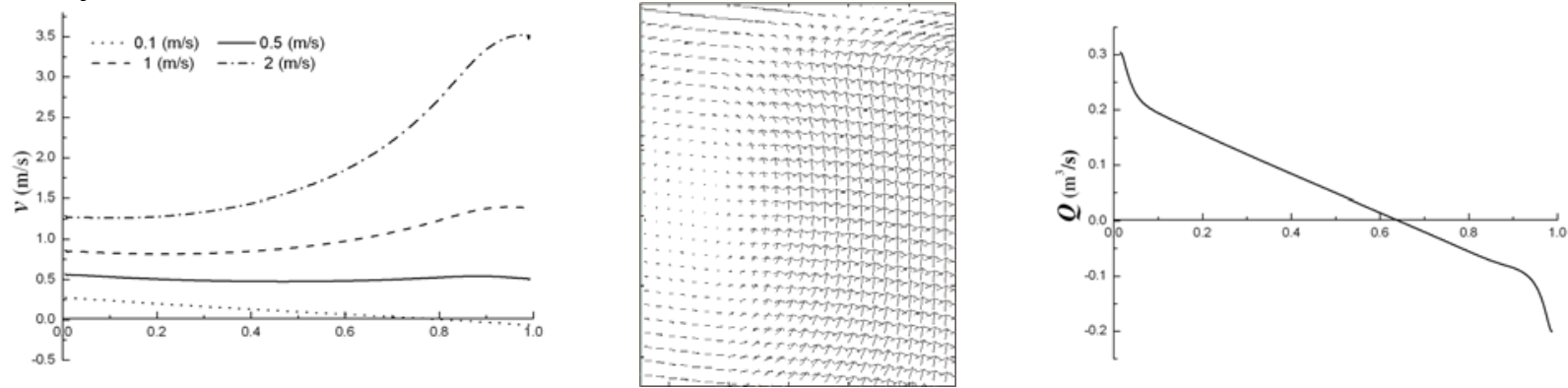

(a) Inlet air velocity distribution

(b) airflow direction of the upper of the system

(c) airflow rate

Fig. 4 Effect of the outlet air velocity on inlet air velocity of the porous solar wall alone the vertical direction

Fig. 5 shows Nusselt number and temperature distribution of the porous solar wall alone the vertical direction at different porosities. It is ob served that the higher porosity of the porous solar wall is, the bigger the Nusselt nu mber is, but the lower the tem perature of the pipe is. It is indicated that is increasing is decreasing at hi gh porosity. So we should choose smaller porosity to get larger heat of the porous solar wall.
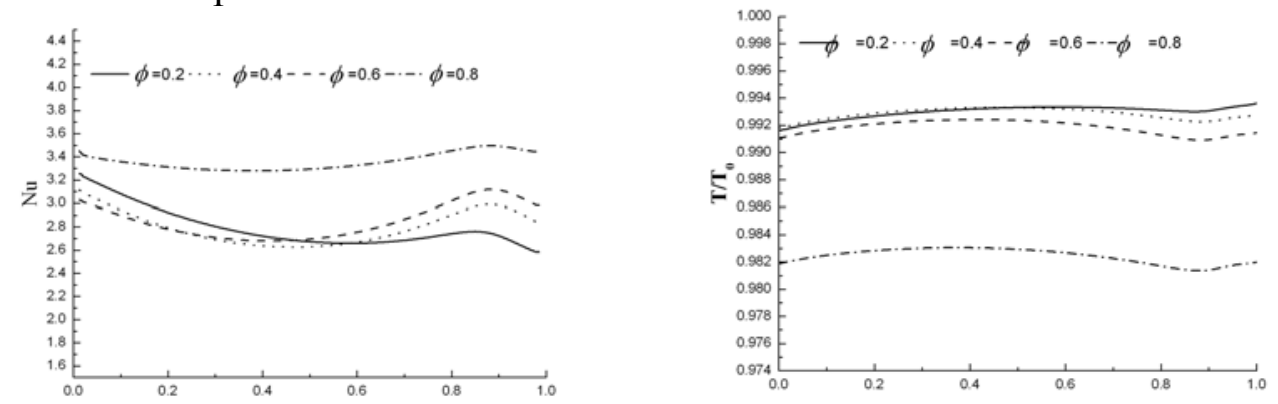

Fig. 5 Effect of porosity on Nusselt number (left) and temperature distribution (right) of the porous solar wall alone the vertical direction.

Fig. 6 shows the porous solar wall width on tem perature distribution of the pi pe alone the vertical direction. With the width increasing, the outlet temperature rising. But as the width further increase, the temperature effect is not obvious. At the sam e time, increasing the width $\mathrm{m}$ eans we should increase the $\mathrm{m}$ aterial consumption and the fan power. In order to reduce the cost of the investment we should choose the proper porous solar wall width.

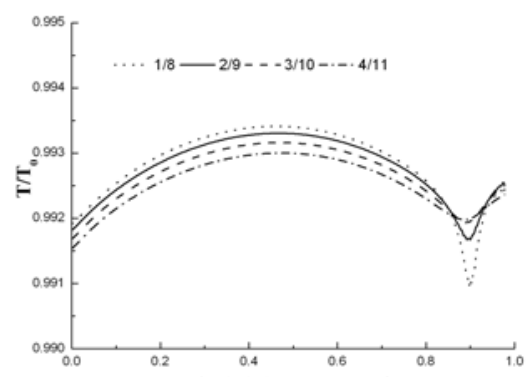

Fig. 6 Effect of the porous solar wall width on temperature distribution of the pipe alone the vertical direction.

\section{Conclusions}


In this paper, the heat transf er and flow characteristics in the porous solar wall system are studied by numerical simulation. It is found that the proposed LBE model is competent of solving the heat and mass transfer in Solar-wall System. The conclusions show that the factors of the porous solar wall s uch as the outle $t$ air v elocity, the porosity a nd porous solar wall width etc., $h$ ave significant effects on th e .Therefore, on the prem ise of dem and for heating, an increas e in the porosity and width of the porous so lar wall, and a decrease the outlet air velocity are advantageous to reduce the operation ener gy consumption. For the real applic ations, the structure of the porous solar wall system should be modified to increase the thermal efficiency and decrease the operation energy consumption.

\section{Acknowledgement}

This work is supported by National Nature S cience Foundation of China under Grant No. 10932010 and 11072220, the National Nature Science Foundation of Zhejiang Province under Grant No. Y607425 and Z6090556, are gratefully acknowledged.

\section{References}

[1] Y. Jiang: Journal of Green Building (2006),p. 10, in Chinese.

[2] D.X. Song: Building energy efficiency design(Tongji unive rcity press,Shanghai 2003), in Chinese.

[3] B.F. Li: Architectural Journal Vol. 11(2001),p. 28. in Chinese.

[4] Z. Yang, X.L. Xu and J.L Han: Acta Energiae Solaris Sinica Vol. 28(2007), p. 1091, in Chinese.

[5] Z.L. Qu: Pass ive solar house building design(China a rchitecture and building press, Beijing (2001), in Chinese.

[6] C.J. Wang, W.J. He and Y.B. Xue: Architectural Journal Vol. 3(2004), p. 76, in Chinese.

[7] S.J. Arulanandam, K. Gtea, D. Hollands and E. Brundrett: Solar energy Vol. 67 (1999), p. 93.

[8] Dymond and Kutscher: A Com puter Design Model for Transpired Solar Co llector Systems, International Solar Energy Conference, American Society of Mechanical Engineers, NewYork, (1995), p.1165.

[9] Hollick: Renewable Energy Vol. 5(1994), p. 415.

[10] Hollick: Renewable Energy Vol. 09(1996), p. 703.

[11] Hollick, Solar Cogeneration Panels, Renewable Energy, 1998. 15: 195-200

[12] W. Chen and W. Liu: Acta Energiae Solaris Sinica Vol. 26(2005), p. 882, in Chinese.

[13] O. Y. Li, L. W ei: characteristics of heat transfer and flow in a new porous heat storage solar wall, Proceedings of the Inaugural US-EU- China Thermophysics Conference, May 28-30, 2009 Beijing, China

[14] W.W. Yan, Y. Liu, Z. L. Guo and Y. S. Xu: International J ournal of Modern Physics C Vol. 17(2006), p.771.

[15] Kutscher, Christensen and Barker: Journal of Solar Engineering Vol. 115 (1993), p. 182.

[16] V. Decker and Hollands: Solar World Congress (1999), p. 248.

[17] J. G. Shao, Y. Liu and Y. S. Xu: Advanced Materials Research Vol. 322 (2011) p. 61.

[18] J. Xu: Housing Science Vol.2 (2006), p. 25.

[19] C.J. Wang, W.J. He and Y.B. Xue: Architectural Journal Vol. 4(2004), p. 24, in Chinese.

[20] C.J. Wang, Y.B. Xue and Y. Yue: Journal of Shangdong Institu te of Arch. and engineering (2004), in Chinese. 